\title{
FRAMEWORK OF MEETING SCHEDULING IN COMPUTER SYSTEMS
}

\author{
Goran Martinovic, Faculty of Electrical Engineering, J.J. Strossmayer University of Osijek, \\ goran.martinovic@etfos.hr
}

\begin{abstract}
Development of computer technologies is a necessary but not the only precondition for solving problems by means of computer supported cooperative work (CSCW). The proposed framework for meeting scheduling encompasses parameters of group members, jobs they carry out, meetings as well as scheduling procedures. In addition to knowledge and experience required for the usage of today's powerful computer technologies, as well as for solving concrete problems, group members, i.e. humans, can be described by a series of individual and sociological properties which can represent an advantage but also a shortcoming of successful CSCW. For the purpose of enabling as successful cooperation intervals, i.e. meetings, as possible, organization, infrastructure and timing parameters of meetings are presented systematically. Jobs carried out at meetings of humans or their resources are adapted to implementation into a greater number of scheduling algorithms. The proposed framework is as such applicable to a wide range of CSCW problems, particularly in the sense of modern technologies and their influence on a human.
\end{abstract}

Keywords: Computer Supported Cooperative Work (CSCW), Group, Human Factors, Meeting, Scheduling.

\section{INTRODUCTION}

World globalization exists in education, economics, research, publishing, entertainment, industry, medicine, resource management and almost all areas of human life. Logically, the importance of knowledge and information, but also the need for cooperation in problem solving has constant progressed. Problems caused in such a huge area appear actually worldwide and are therefore much more complicated than the problems produced or supposed to be solved by a human as an individual. The support comes in the form of global means of computer systems, communication and networking on all levels and especially the Internet, www, mobile communication and computational grids. Real-time problems are rather extensive, particularly in case of hard deadlines. A human represents an active component in Computer Supported Cooperative Work (CSCW) and requires special attention.

Organizations of any form reach their goals by using computer technologies and tools enabling networking of individuals and groups in a cooperative unit. According to [19], that term is known as CSCW. Various terms have been used for CSCW, such as workgroup computing, computer-assisted communication, computer supported groups, etc. According to [10], CSCW is a generic term which combines understanding of the way people work in groups with enabling technologies of computer networking, and associated hardware, software, services and techniques. CSCW implies a constant flow of knowledge and experience of group members, communication which enables flexibility and redundancy, teamwork above individual work, as well as the environment in which members cooperate. The environment is represented by the work the group is carrying out.

CSCW is also observed through the term groupware. It includes specialized computer aids designed for the use of collaborative work groups and it frequently involves software, hardware, services and/or group support. Groupware are computer-based systems that support groups of people engaged in a common task and provide an interface to a shared environment.

Regardless of the development of computer and especially networking technologies, a time-critical or real-time problem remains interesting. The reason for that is that computers, software support and communication are used in CSCW as a support to cooperative work, while group members and work being carried out by them represent scheduling factors. The main purpose of this paper is to include all significant organization and timing parameters of CSCW through the proposed meeting scheduling model. The model is focused on the properties of group members meetings at which they cooperate and do their job. Special attention is given to those group members that possess both knowledge and capabilities, but also to shortcomings of team work. The model should provide a possibility of a better CSCW factor estimation and setting of corresponding timing demands on the work being carried out by the group. Parameters comprised by the model enable 
virtualization of a human-computer interface. Regardless of the model assuming the group members are computer-, software- and communication-supported, it can be applied in the cases when group members are computers with humans being exclusively external users, as presented in [22].

CSCW and its basic principles are described in the introductory part of this paper. The second chapter gives a time-space classification of CSCW systems, reasons for their usage as well as examples of asynchronous and synchronous applications. The third chapter describes the scheduling problem in CSCW, whereas the fourth one presents the meeting scheduling model in CSCW. The paper concludes with a short analysis of the proposed model and implementation features.

\section{CSCW APPLICATIONS}

According to [21], CSCW technology is basically classified with respect to the place and time of group members' work. If group members work together at the same time, we talk about a synchronous CSCW [13]. Otherwise, the CSCW is asynchronous. Regarding a working location, group members can work collocated or "face-to-face" group. On the other hand, we differ non-collocated or distance groups. Location and time have a very important influence on group functionality, formation and work. CSCW has more positive consequences. It can enable a faster, efficient and transparent communication [7], reduce working costs, expand horizons of group members regarding problem understanding and solving, simplify group coordination, improve interaction, as well as usability and availability of computer systems [15].

In the next chapters well known asynchronous and synchronous applications of CSCW technology are described.

\section{Asynchronous CSCW}

Most popular asynchronous applications are e-mail (including mailing lists), news groups, hypertext, blogs, facebooks, etc. They enable an exchange of different forms of information between two and more persons or groups of persons. Multiple accesses to a www and other forms of documents and information are fundamental properties of these applications [12]. Group calendars enable scheduling, project leading and coordination between a greater number of people.

\section{Synchronous or real-time CSCW}

Shared whiteboards enable two or more users to see and draw on the shared whiteboard from different locations. The location at which every application participant draws which color he or she uses, etc. is known at any instant. Video communication enables a fast video link. In addition to mutual conversation, it enables cooperation in various situations through observing activities at a distant location. Different chat systems enable more people to write and read messages in textual or visual (video) form and exchange them in real-time at some public place. Thereby it is possible to realize the access control, introduction of the moderator leading a discussion, proposal of discussion topics, but also an entirely free and sporadic communication. Decision support systems contain tools for brainstorming, criticizing of ideas, associating weights and possibilities to events, and voting. Very good examples of a real-time application are multi-player games, accessed mostly through the Internet. The problem of real-time operating depends on a variety of CSCW properties, as in [18]. Some of them are encompassed by the proposed model.

According to [14], due to the presence of very fast computer networks, powerful computational clusters and grids, wireless and mobile technologies in all aspects of human life, the aforementioned applications have faced new ways of application with new requests being placed as well.

\section{CSCW SCHEDULING}

Cooperation effectiveness of group members significantly prescribes group effectiveness. That cooperation is targeted to better understanding of tasks, efficient resource management and development of cooperation strategy. Successful groups support mutual respect of members, include all available knowledge in the cooperation process [7], carry out careful resource sharing and open different viewpoints regarding the given problem and their recognition [6]. Group members acquire new knowledge and experience in time, get used to each other and adjust to the cooperation process. Since the group possesses certain knowledge and experience, can make decisions and is computer supported, we talk about artificial intelligence of the group.

According to [15], the scheduling problem can be described as matching tasks on processor(s) and sharing of processor time in the assigned time slots. Scheduling in the CSCW system is more complex. 
Group members cooperate in order to carry out a certain job. Time intervals when they cooperate are called a meeting. During the meeting, members use available computer and network resources and cooperate. Scheduling of meetings means that jobs are scheduled to group members in solving the given tasks. Only some of the questions that should be answered by meeting scheduling are: meeting time, meeting duration, meeting participants, meeting place, etc.

Since there is no optimal scheduling algorithm for solving all situations, for this problem we use artificial intelligence procedures similar to [17] and heuristic procedures as in [11]. Intelligent schedulers must be familiar with interests and priorities of possible group members supposed to be met. Calendar schedule of every person taking part in the meeting is forwarded to his/her local scheduler, who makes the main meeting schedule. Most often schedulers work concurrently in order to shorten scheduling time, increase reliability concerning calendar interpretation and enable protection of private interests. Local schedulers must also cooperate and exchange information about meetings. Most frequently the scheduler system is based on one principal and a series of local schedulers. Scheduling procedure autonomy requires a detailed definition of the meeting goal, group champion selection among group members, as well as support of various user and interaction types, as described in [23].

\section{MEETING SCHEDULING IN CSCW}

Similarly to [16], the CSCW system can be presented by group ( $G$ ), job trusted to group $(J$ ), cooperation $(C)$ and scheduling $(S)$. Parameters of all levels are listed in alphabetical order in Appendix.

\section{Group}

Group $G$ consists of $n$ members. Every member $M_{i}$ of group $G$ can be shown by (1):

$$
M_{i}=\left\{A_{M i}, \varepsilon_{i}, s_{i}, \varpi_{i}, \omega_{i}, \operatorname{distr}_{i}, I_{i}, C S_{i}, h s p_{i}\right\}
$$

Group member parameters are described in the text to follow.

$A_{M i}$ - member availability according to the schedule. A personal schedule of a member consists of an ordered set of points in time bounding schedule timing gaps. These points build the calendar schedule. Every point contains a datum on the exact date and time. $\varepsilon_{i}$ - experience level of a group member. It corresponds to the time spent in the group or on similar jobs.

$s_{i}$ - special knowledge level of a group member. It depends on the time and success spent on special educations, workshops or similar activities related to group activities.

$\varpi_{i}$ - personal interest level of a group member. For the group efficacy, the level of personal interest should be as low as possible. On the other hand, it must not be lower than the sociological level ${ }^{S o c_{M}}$ which motivates a member to participate in group work, similarly to [4]. According to (2), that sociological level is determined by the level of anticipated reciprocity a member may expect from the group ( $r e c$ ), expected increased reputation (rep), sense of efficacy ( $e f f$ ), and sense of community ( comm ).

$$
\mathrm{soc}_{M}=(\text { rec, rep, eff }, \mathrm{comm})
$$

$\omega_{i}$ - general interest level of a group member. It should be as high as possible.

distr $_{i}$ - the law of statistical distribution of group member availability or an availability sample. It is especially important in case of sporadic requests for meetings. It is obtained by a statistical analysis of availability time.

$I_{i}$ - group member infrastructure level. A member with a better infrastructure, i.e. better computer support and communication towards the group, as in [12] and [1], is more easily incorporated into the group. For the purpose of model simplicity, an equal infrastructure level is sometimes assumed for all members. In real life, the situation is completely different; it is the group member infrastructure which is the most obvious indicator of member heterogeneity. Internal ordering of the group member infrastructure depends on the so-called platform parameters shown in [15] and by (3). These parameters differ as computer (comp) and communication ( com ) parameters.

$$
I=\left\{\begin{array}{c}
\text { comp-OS, arch, clk, } k, \text { mem, hdd } \\
c o m-n e t, I C N, \text { mob }, n_{\text {ICN }}, s_{\text {cluster }}, \mathrm{com}^{2}, \\
X_{\text {spt }}, X_{\text {conc }}
\end{array}\right\} \text { (3) }
$$

Computer parameters are platform operating system ( $O S$ ), machine architecture (arch), processor speed 
(clk), number of processors in machine $(k)$, memory size (mem) and storage or disk space ( hdd ). Communication parameters are nominated network speed (net), interconnection network topology ( ICN ), member mobility - members can be mobile if they are connected by a mobile [14] or a wireless network [20], whereby a higher level of mobility is naturally offered by a mobile network $(m o b)$, number of arbitrary interconnections $\left(n_{\text {ICN }}\right)$, cluster size ( $s_{\text {cluster }}$ ), overlapped computation/ communication $\left(\mathrm{com}^{2}\right)$, external support to machine $\left(X_{\text {spt }}\right)$ and machine capability of resource concession $\left(X_{\text {conc }}\right)$.

$C S_{i}$ - additional credits given to a member by the host or group champion members.

$h s p_{i}$ - human standpoints of a group member. Although there are many human standpoints, according to (4), they can be reduced to the ethical, cultural, religious and political level.

$$
\text { hsp }=\{e t h, c u l, r e l, p o l\}
$$

The goal of the meeting may to a certain degree be inconsistent with the given principles of the group member. According to [9], ethical principles may imply social, environmental, scientific and other principles. It is desirable to have a group member of the highest cultural level possible, since it is favorable to cooperation with other group members as well as the group as a whole. A member can be expected not to participate in the meeting below a targeted cultural level. This also holds for religious and political standpoints. Members are expected to be religiously and politically tolerant, but they cannot be forced into participation in the meeting which significantly disagrees with their religious or political orientation.

In contrast to other group member parameters, human standpoints represent a completely inalienable right of every member not obliged to subject them to group interests. On the other hand, the group may be more or less supportive of those principles. If standpoints of the group and the member differ significantly, it would be most reasonable for both sides to break off their relations.

According to [1], a group is said to be heterogeneous if its members mutually differ in at least one of the given parameters. In real life, group members may be expected to differ in a greater number of parameters. It would be difficult to show heterogeneity for all members in all parameters, and if it were shown, it would be almost useless in case of meeting scheduling. Therefore, it is expressed by a group performance indicator for every member $M_{i}$ of group $G$, which is denoted by $H_{M_{i}}$. That group indicator, according to expression (5), corresponds to the mean value of all parameters par $_{k}^{M_{i}}$ of $k=1,2, \ldots x$ for some member $M_{i}$. According to (6), group indicators $H_{M_{i}}$ placed in vector $H_{G}$ show heterogeneity among members of group $G$.

$$
\begin{aligned}
& H_{M_{i}}=\sum_{k=1}^{x} \frac{\operatorname{par}_{k}^{M_{i}}}{x} \\
& H_{G}=\left\lfloor H_{M_{1}}, H_{M_{2}}, \ldots, H_{M_{n}}\right\rfloor
\end{aligned}
$$

Representation of group heterogeneity in a greater number of parameters would require a matrix notation of $H_{G}$.

\section{Jobs}

Job $J$ that should be carried out by the group consists of $m$ tasks $T_{1}, T_{2}, \mathrm{~K}, T_{m}$. Every task can be presented by (7). Some of the task parameters follow from [17].

$$
T_{j}=\left(c_{j}, t_{d j}, p_{j}, t_{i a j}, \text { hard / soft, pmtn, dupl }\right)
$$

where:

$c_{j}$ - execution time.

$t_{d j}$ - deadline.

$p_{j}$ - period for periodic task.

$t_{i a j}$ - interarrival time for aperiodic task.

hard/soft - type of deadline.

pmtn - possibility of terminating the task during execution (preemptability).

dupl - possibility of task duplication.

Similarly to the group, job $J$ can also be heterogeneous, since tasks making it do not have to be mutually equal. In contrast to group heterogeneity, heterogeneity of job $J$ is expressed by some of the most important individual indicators for every task $T_{j}$. That vector $H_{J}$ contains values of these individual parameters $H_{T_{j}}$, and specifically in (8) execution time is in the worst case $c_{j}$. 


$$
H_{J}=\left[c_{1}, c_{2}, \ldots, c_{m}\right]
$$

Representation of job heterogeneity in a greater number of parameters would require a matrix notation. Studying heterogeneity should guarantee a meeting schedule of high quality, such that a member of a certain heterogeneity level (group indicator values of its performances) will be associated a job of a certain heterogeneity level (of certain duration, requests on resources, etc.).

\section{Cooperation intervals}

Members of a group that execute a job or a series of tasks must cooperate. Cooperation intervals are called meetings $(C)$. The scheduling procedure boils down to scheduling jobs to group members, i.e. filling in their schedule by meetings.

The $k$-th meeting or cooperation $C_{k}$ can be shown as in (9), where $\rho$ represents organizational and infrastructural properties, $\delta$ timing properties of the meeting and hsp human standpoint properties. Expression (10) represents parameters $\rho$.

$$
C_{k}=\left\{\rho_{k}, \delta_{k}, h s p_{k}\right\}
$$

$\rho_{k}=\left\{G M_{k}, \chi_{k}, \operatorname{org}_{k}, \varepsilon_{k}, s_{k}, \varpi_{k}, I_{k}, \varphi_{k}, \operatorname{cham}_{k}\right\}(10)$ where:

$G M_{k}$ - group members included in the job execution (meeting). It is obtained by selecting members for a certain job and scheduling jobs. Selection criteria are carried out according to some of the elements describing them. A member is selected who is available in the requested interval, possesses a certain level of experience, special knowledge, general interests and a satisfactory level of infrastructure. A set of group members must be defined prior to scheduling, but it can be substituted during meeting (by eliminating inadequate members and involving members of higher quality).

$\chi_{k}$ - meeting hosts must be defined prior to scheduling, but it can be altered during the meeting, also for the purpose of improving the quality of hosts. A host can be a group member, a group member with a special status, or a rented member. A host is better if he/she belongs to the group and agrees with general or common interests of the group.

$\operatorname{org}_{k}$ - estimated level of meeting organization. It should be as high as possible, as required in [21]. Estimation is done either by the hosts or group members. Before the meeting, $\operatorname{org}_{k}$ makes participants aware of the importance of the forthcoming meeting. After the meeting, it should clear organizational shortcomings of future meetings. Further sections give parameters describing expected levels of experience, knowledge, interest and infrastructure properties of potential group members. They are used for recruitment of members into the group by comparing parameters of a member that would like to join the group with a boundary value. A boundary value is often an average parameter value.

$\varepsilon_{k}$ - expected experience level of group members is a minimal experience level, which should be satisfied by the group member joining the group.

$s_{k}$ - expected level of special knowledge necessary at the meeting. It corresponds to the minimal value of the special knowledge level that a member should have in order to join the group.

$\varpi_{k}-$ allowed level of personal interests of group members. For some member, it must be greater than or equal to the allowed, i.e. average value.

$I_{k}$ - level of infrastructure ordering of a potential group member $I_{i}$.

$\varphi_{k}$ - meeting priority level. It should be known before the start of invitation of potential members for a meeting. It is determined by hosts of the meeting.

cham $_{k}$ - number of champions in the meeting. Their task in future meetings is to recruit new group members.

Expression (21) shows meeting timing parameters.

$\delta_{k}=\left\{t_{c k}, \Delta t_{c k}, c_{k}, t_{s k}, t_{d k}, t_{D k}, p_{k}, t_{i a k}\right\}$

where:

$t_{c k}$ - meeting duration. Time planned for a successful end of the meeting, i.e. job. It depends on the organizational level and some other parameters.

$\Delta t_{c k}$ - meeting prolongation time. It is a reserve time for a possible prolongation of the meeting. It depends finally on job complexity and the meeting organization.

$c_{k}$ - real duration of meeting.

$t_{s k}$ - meeting start time.

$t_{d k}$ - meeting deadline. 
$t_{D k}$ - decision time in which the meeting must be accepted or canceled.

$p_{k}$ - the period in which the meeting is carried out.

$t_{i a k}$ - interarrival time between consecutive meetings of the same groups.

Meeting human standpoints parameters $\left(h s p_{k}\right)$ are shown by (4). These parameters can significantly influence group cooperation, which will mostly be manifested through group member availability. Since members cannot influence human standpoints of the group, the group should not pose them as a direct criterion of involving members into meeting. On the other hand, inappropriate human standpoints of the host or champion members should result in removal of their favorable status in the group.

\section{Scheduling}

Scheduling is a procedure of scheduling available computer and communication resources or capacities for the tasks being executed. Created time schedule determines the time when the task approaches the resources. The most scheduled resource in the computer system is a processor - one or more of them. Numerous scheduling schemes presented in [3] have been developed that are based on analytical or heuristic procedures. A scheduling result can be estimated through satisfaction of hard timing requests with acceptable utilization.

Meeting scheduling in CSCW also represents resource scheduling to users [16]. However, resources represent a job to be done, and the users of these resources are group members. Group members can also be users of the job being executed by the group. In order to carry out CSCW, group members must cooperate, i.e. they must have a meeting. These meetings can be carried out as direct group member meetings, thus becoming meetings of all resources or goods they have at their disposal; whereby we have in mind computer resources, bases of knowledge or goods which can be used for general interest of the group doing computer supported cooperative work. Basic scheduling procedure parameters are given by expression (12):

$$
S=\left\{\begin{array}{l}
\gamma, T_{\text {dep }}, c_{S}, p^{2} \operatorname{tn}_{S}, \text { reS }, \text { al }, T_{\text {dupl }}, \\
L_{S}, F T, H_{S}, S_{\text {static }}^{\text {dynamic }}, \text { auction }
\end{array}\right\}
$$

where:

$\gamma$ - objective function or an optimal scheduling criterion. According to [3], for individual tasks those are execution time, flow time, latency, delay, earliness, whereas for a set of tasks of the schedule as a whole those would be makespan, mean flow time, mean weighted time, maximum latency and utilization.

$T_{\text {dep }}$ - task dependency. The scheduling problem of mutually dependent tasks is much harder than the scheduling problem of mutually independent tasks whose schedule referring to job execution is not important.

$c_{S}$ - expected or achieved scheduling duration. It is the time planned for solving some scheduling problem, most often strictly limited, so that the choice of algorithms becomes restricted to low complexity algorithms executable in polynomial time. Recent literature, as in [2], presents a lot of NPhard scheduling problems, so that in addition to the so-called pure algorithm approach, a series of relaxation conditions, approximation algorithms, as well as rules for scheduling and heuristic procedures is introduced.

$\mathrm{pmtn}_{S}$ - scheduling preemption. This is one of the relaxation criteria implying that the scheduling procedure itself can be interrupted by some other activity or some other scheduling procedure in the environment.

reS - rescheduling mechanism. According to [8], rescheduling represents a dynamic procedure of renewing the existing schedule as a response to significant disorders or changes whereby the information on possible future system operation is also taken into consideration. Rescheduling mechanisms may significantly improve both resource management and meeting scheduling.

al - scheduling algorithms used. Numerous deterministic and stochastic scheduling algorithms are described in [17]. But, only some are directly applicable in meeting scheduling.

$T_{\text {dupl }}$ - task multiplication/duplication, which is linked to parameter dupl in expression (7).

$L_{S}$ - location of a scheduler. The scheduling procedure itself can be carried out at one location (centralized). Centralized scheduling is mostly done under supervision, i.e. it is arranged by the job (application) host. Decentralized scheduling is entrusted with the best group members.

FT - fault tolerance implemented in the scheduling procedure. The goal is to increase reliability and availability of meetings, but it mostly reduces to redundancy increase. 
$H_{S}$ - acceptable environment heterogeneity by the scheduling procedure. Regardless of the fact that environment heterogeneity increases scheduling complexity, according to [1], jobs consisting of various tasks can be done very successfully by various group members. Hence, an appropriate scheduling algorithm is necessary, which would make the best possible matching, i.e., scheduling, at the group level $\left(H_{M i} \leftrightarrow H_{T j}\right.$, with the matching direction depending on the applied scheduling algorithm).

$S_{\text {static }}^{\text {dynamic }}$ - timing properties of scheduling execution. According to [15], it can be static, dynamic or combined (static-dynamic). Best scheduling is naturally expected from static-dynamic scheduling procedures. After the initial static schedule, if necessary, during schedule execution (dynamic) they can change the schedule.

auction - possibility of auction within the scheduling procedure. According to [5], auction means that group members can bid jobs or tasks to be carried out. In contrast to a classical evaluation of group members on the basis of which they are assigned jobs by the host or champion, bidding, i.e. fight for jobs, is run directly among members. It is based upon offering better conditions under which jobs of the group are to be done.

The meeting scheduling procedure is based upon the previously described model. According to the function, parameters of the described model can be classified into organizational and timing, and from the scheduling point of view, they can be internal and external, as well as input and output. Internal parameters set conditions in which scheduling is done, whereas the external ones are represented by the interaction with environment, i.e. the group. Figure 1 presents the CSCW meeting scheduling procedure. In this paper the lowest scheduling level are the meetings. We can also do scheduling at the lower level of mutually dependent tasks, as well as schedule them to group members. This takes place more often if group members are referred to as computers, not computer supported human work.

Successful scheduling implies involving as many parameters of the described model as possible into the scheduling criteria. The procedure is based on several basic assumptions and steps:

1. Define job $J$ that should be executed by $G$.
2. Define hardness of timing requests, which depends on the application - synchronous or asynchronous.

3. Define the initial parameters of available members $M$ for group forming.

4. Select group members according to organizational, i.e. infrastructural properties of the group from set $\rho_{k}$ and the properties of members from $M_{i}$.

5. Select the timing parameter or parameters from set $\delta_{k}$ according to which meeting scheduling will be executed $c_{k}$. For a greater number of parameters, scheduling is iterative and multilayered.

6. Implement a scheduler on local and/or the level into CSCW.

7. By applying the criteria described in step 4, carry out scheduling $S$ and make a calendar schedule of cooperation $K$.

8. Apply evaluation criteria of scheduling.

9. Make scheduling corrections (during a dynamic phase or a rescheduling procedure).

Figure 1. Meeting Scheduling Procedure

\begin{tabular}{|c|c|c|}
\hline \multicolumn{3}{|c|}{\begin{tabular}{|l}
$S$ \\
$\gamma, T_{\text {dep }}, c_{S}, p m t n_{S}$, reS, al, $T_{\text {dupl }}$, \\
$L_{S}, F T, H_{S}, S_{\text {static }}^{\text {djnamic }}$, auction
\end{tabular}} \\
\hline \multirow[t]{11}{*}{$C$} & \multicolumn{2}{|c|}{$C_{1}, C_{2}, \ldots, C_{k}$} \\
\hline & $\rho_{k}$ & $G M_{k}, \chi_{k}, \operatorname{org}_{k}, \varepsilon_{k}, s_{k}, \varpi_{k}, I_{k}, \varphi_{k}$, cham $_{k}$ \\
\hline & $\delta_{k}$ & $t_{c k}, \Delta t_{c k}, c_{k}, t_{s k}, t_{d k}, t_{D k}, p_{k}, t_{i a k}$ \\
\hline & $h s p$ & eth,cul,rel,pol \\
\hline & \multirow[t]{4}{*}{$J$} & $H_{I}$ \\
\hline & & $T_{1}, T_{2}, \ldots, T_{m}$ \\
\hline & & $c_{j}, t_{d j}, p_{j}, t_{\text {iaj }}$, hard / soft, pmtn, dupl \\
\hline & & $\hat{\imath}$ \\
\hline & \multirow[t]{3}{*}{ G } & $H_{G}$ \\
\hline & & $M_{1}, M_{2}, \ldots, M_{n}$ \\
\hline & & $A_{M i}, \varepsilon_{i}, s_{i}, \bar{w}_{i}, \omega_{i}$, distr $_{i}, I_{i}, C S_{i}, h s p_{i}$ \\
\hline
\end{tabular}

Almost all timing parameters can be the key to scheduling. Then scheduling boils down to sorting according to a relevant parameter and forming of an execution queue. For example, meetings like a meeting whose deadline is shorter, or a meeting that has the shortest real duration time, or a meeting with the highest initial priority, etc. can be prioritized. 
Extensive explanation of the scheduling procedure is not given in this paper.

\section{CONCLUSIONS}

Increased throughput of computer networks, Internet technology and mobile communications contributes significantly to availability of powerful computer resources, but also to a mutual connection between humans. Besides asynchronous CSCW applications, this has enabled development of numerous synchronous CSCW applications with hard timing requests, such as telemedicine, decision making in production processes, and control of computer, but also natural, resources.

The proposed framework of meeting scheduling takes into account a great number of general CSCW parameters, but also parameters of the group and its members, jobs they jointly do in meeting intervals, as well as properties of these meetings. The framework also describes a possibility of implementing the given parameters into the scheduling procedure. Although it relies on the scheduling theory and scheduling of computer resources, the paper focuses on the human. General and specific knowledge of a human, his/her care directed towards their own computer infrastructure, the level of adaptability of personal interests to group interests, as well as sociological understanding of cooperation among group members, are the key to successful cooperation in problem solving. Organizational/infrastructural and timing parameters of meetings are of special importance, since on the basis of them it is possible to organize a meeting in accordance with a corresponding scheduling procedure. The advantage of the proposed approach is a full integration into new computer and communication technologies, but also a sociological viewpoint adapted to these technologies, which does not neglect human dis/advantages.

The proposed framework for scheduling meetings in CSCW is a result of a years-long application and modification of the described principles used in handling resources in distributed computer systems, in which it happens very often that a human represents the weakest link as to problem solving through cooperation. Current and future research deals with further application and evaluation of models in mobile and grid environments, which will definitely result in many new dimensions of CSCW, especially of a human in these new technological conditions.

\section{REFERENCES}

1. Bharadway V., \& Reddy Y.V. (2003). A framework to support collaboration in heterogeneous environment, ACM SIGGROUP Bulletin, 24(3), 103-116.

2. Blazewicz J., Kovalyov M.Y., Machowiak M., Trystram D., \& Weglarz J. (2006). Preemptable Malleable Task Scheduling Problem. IEEE Transactions on Computers, 55(4), 486-490.

3. Brucker P., Dhaenens-Flipo C., Knust S., Kravchenko S.A., \& Werner F (2000). Complexity Results for Parallel Machine Problems with a Single Server, Journal of Scheduling, 5, 429-457.

4. Carayon P. (2006). Human factors of complex sociotechnical systems, Applied Ergonomics, 37, Elsevier, 525-535.

5. Chen J., Chen X., Kauffman R.J., \& Song X. (2006). Cooperation in Group-Buying Auctions, Proc. of $39^{\text {th }}$ Annual Hawaii International Conf. on System Sciences (HICSS'06), 121c.

6. Chen I.Y.L., Su A., Huang J., Lan B., \& Shen Y.S. (2006). Ubiquitous collaborative learning in knowledge-aware virtual communities, Proc. of IEEE International Conf. on Sensor Networks, Ubiquitous and Trustworthy Computing (SUTC'06), 84- 89.

7. Cogburn D.L., Zhang L., \& Khothule M. (2002). Going global, locally: the socio-technical influences on performance in distributed collaborative learning teams, ACM Int. Conf. Proc. Series, 30, 2002 Annual Research Conference South African Inst. Computer Scientific Information Technology Enablement through Technology, 52-64.

8. Viera G.E., Herrmann J.W., \& Lin E (2003). Rescheduling Manufacturing Systems: A Framework of Strategies, Policies, and Methods, Journal of Scheduling, 6(1), 39-62.

9. Henrich J., \& Henrich N. (2006). Culture, evolution and the puzzle of human cooperation, Cognitive Systems Res., 7, Elsevier, 220-245.

10. Kamsin A., \& Sabri M.I.M. (2004). The collaborative system strategies, Proc. 2004 ACM Int. Symp. on Information and Communication Technologies, 158-165.

11. Kozierok R., \& Maes P. (1993). A learning interface agent for scheduling meetings, Proc. of $1^{\text {st }}$ ACM Intelligent User Interfaces, 81-88.

12. LeJeune N. (2003). Critical components for successful collaborative learning in CS1, Journal 
of Computer Supported Collaboration, 19(1), 275285.

13. Li D., Zhou L., \& Muntz R.R. (2000). A New Paradigm of User Intention Preservation in RealTime Collaborative Editing Systems, Proc. of $7^{\text {th }}$ IEEE Int. Conf. on Paralell and Distributed Systems (ICPADS'00), 401-408.

14. Looney C.A., \& Valacich J.S. (2005). Mobile technologies and collaboration, Proc. $38^{\text {th }}$ IEEE Ann. Hawaii Int. Conf. System Sciences, 31.

15. Martinovic G. (2003). Owner/User Role in Computational Grid Extension by Non-dedicated Resources, Proc. ACM Int. Symp. on Supporting Group Work (Group'03), 221-228.

16. Martinovic G., \& Budin L. (2002). Real-Time Meeting Scheduling Model by Computer Supported Cooperative Work, Intelligent Information Processing, Kluwer, 289-292.

17. Pinedo M. (2001). Scheduling: Theory, Algorithms, and Systems ( $2^{\text {nd }}$ Ed.), PTR.

18. Rinkus S., Walji M., Johnson-Throop K.A., Malin J.T., Turley J.P., Smith J.W., \& Zhang J. (2005). Human-centered design of a distributed knowledge management system, Journal of Biomedical Informatics, 38, Elsevier, 4-17.

19. Schmidt K. \& Simone C. (2000). Mind the gap! Towards a unified view of CSCW, Proc. of $4^{\text {th }}$ Int. Conf. on Design of Cooperative Systems (COOP'00), 205-221.

20. Sumiya T., Inoue A., Shiba S., Kato J., Shigeno H., \& Okada K. (2004), A CSCW system for distributed search/collection tasks using wearable computers, Proc. $6^{\text {th }}$ IEEE Workshop Mobile Computing Systems and Applications (WMCSA'04), 20-27.

21. Tjosvold D., West M.A., \& Smith KG (2003). Teamwork and cooperation, Fundamentals of organizational effectiveness, Int. Handbook of Organizational and Cooperative Working, John Wiley \& Sons, 3-8.

22. Tolone W., Ahn G.J., Pai T., \& Hong S.P. (2005). Access control in collaborative systems, ACM Computing Surveys, 37(1), 29-41.

23. Zhuge H. (2002). A knowledge grid model and platform for global knowledge sharing, Expert Systems with Appl., 22(4), Pergamon, 313-320.

This work is supported by research project grant No. 165-0362980-2002 from the Ministry of Science, Education and Sports of the Republic of Croatia.

\section{APPENDIX}

Table 1. Parameter Names

\begin{tabular}{|c|c|}
\hline \multicolumn{2}{|r|}{ Parameter } \\
\hline Symbol & Name \\
\hline \multicolumn{2}{|r|}{ G - Group } \\
\hline arch & Machine architecture \\
\hline$\overline{A_{M i}}$ & Member availability \\
\hline clk & Processor speed \\
\hline com & Communication parameters \\
\hline comm & Sense of community \\
\hline comp & Computer parameters \\
\hline $\mathrm{com}^{2}$ & $\begin{array}{l}\text { Overlapped computing/ } \\
\text { communication }\end{array}$ \\
\hline$C S_{i}$ & Additional credits to members \\
\hline eff & Sense of efficacy \\
\hline hdd & Disk space \\
\hline$h s p_{i}$ & $\begin{array}{l}\text { Human standpoints (ethical, } \\
\text { cultural, religious, political) }\end{array}$ \\
\hline$H_{M_{i}}$ & Group heterogeneity \\
\hline$I_{i}$ & Infrastructure level \\
\hline$I C N$ & Interconnection network \\
\hline$k$ & Number of processors \\
\hline mem & Memory size \\
\hline$m o b$ & Member mobility \\
\hline$M_{i}$ & Group member \\
\hline net & Network speed \\
\hline$n_{I C N}$ & Arbitrary interconnections \\
\hline OS & Operating system \\
\hline rec & Anticipated reciprocity \\
\hline rep & Expected reputation \\
\hline$S_{\text {cluster }}$ & Cluster size \\
\hline$s_{i}$ & Special knowledge level \\
\hline$S O C_{M i}$ & Sociological level \\
\hline$X_{\text {conc }}$ & $\begin{array}{l}\text { Machine capability of resource } \\
\text { concession }\end{array}$ \\
\hline$X_{s p t}$ & External support to machine \\
\hline$\varepsilon_{i}$ & Experience level \\
\hline$\omega_{i}$ & General interest level \\
\hline$\varpi_{i}$ & Personal interest level \\
\hline \multicolumn{2}{|r|}{$J-$ job } \\
\hline$c_{j}$ & Execution time \\
\hline dupl & Task duplication \\
\hline
\end{tabular}




\begin{tabular}{|c|c|}
\hline Symbol & Name \\
\hline pmtn & Task preemptability \\
\hline$t_{d j}$ & Task deadline \\
\hline$t_{i a j}$ & Interarrival time for aperiodic task \\
\hline hard/soft & Type of deadline \\
\hline$H_{J}$ & Job heterogeneity \\
\hline$p_{j}$ & Period for periodic task \\
\hline$T_{j}$ & Task \\
\hline \multicolumn{2}{|r|}{$C$ - cooperation interval } \\
\hline$C_{k}$ & Real meeting duration \\
\hline $\operatorname{cham}_{k}$ & Number of champions \\
\hline$G M_{k}$ & $\begin{array}{c}\text { Group members included in the job } \\
\text { execution }\end{array}$ \\
\hline$I_{k}$ & Infrastructure ordering \\
\hline $\operatorname{org}_{k}$ & Level of meeting organization \\
\hline$p_{k}$ & Meeting period \\
\hline$S_{k}$ & $\begin{array}{l}\text { Expected level of special } \\
\text { knowledge }\end{array}$ \\
\hline$t_{i a k}$ & Meeting interarrival time \\
\hline$t_{s k}$ & Meeting start time \\
\hline$t_{c k}$ & Meeting duration \\
\hline$t_{d k}$ & Meeting deadline \\
\hline
\end{tabular}

\begin{tabular}{|c|c|}
\hline Symbol & Name \\
\hline$t_{D k}$ & Decision-accept or cancel meeting \\
\hline$\delta$ & Timing properties \\
\hline$\varepsilon_{k}$ & Expected experience level \\
\hline$\chi_{k}$ & Meeting host \\
\hline$\varphi_{k}$ & Meeting priority level \\
\hline$\varpi_{k}$ & $\begin{array}{c}\text { Allowed level of member personal } \\
\text { interests }\end{array}$ \\
\hline$\Delta t_{c k}$ & Meeting prolongation time \\
\hline \multicolumn{2}{|r|}{$S$ - scheduling } \\
\hline al & Algorithm \\
\hline auction & Possibility of auction \\
\hline$c_{S}$ & Scheduling duration \\
\hline$F T$ & Fault tolerance \\
\hline$H_{S}$ & $\begin{array}{c}\text { Acceptable environment } \\
\text { heterogeneity }\end{array}$ \\
\hline$L_{S}$ & Location of scheduler \\
\hline pmtn $_{S}$ & Scheduling preemption \\
\hline reS & Rescheduling mechanism \\
\hline$S_{\text {static }}^{\text {dynamic }}$ & Timing properties of scheduling \\
\hline$T_{\text {dep }}$ & Task dependency \\
\hline$T_{\text {dupl }}$ & Task multiplication/duplication \\
\hline$\gamma$ & Objective function \\
\hline
\end{tabular}

